\title{
Conceptual Optimization of a Generalized Net Model of a Queuing System
}

\author{
Velin Andonov
}

\author{
Stoyan Poryazov
Mathematics and Inte of \\ Bulgarian Academy of Sciences \\ Acad. G. Bonchev Str, block 8 \\ 1113 Sofia, Bulgaria \\ Email: stoyan@math.bas.bg
}

\author{
Emiliya Saranova
}

Institute of Mathematics and Informatics

Bulgarian Academy of Sciences

Acad. G. Bonchev Str, block 8

1113 Sofia, Bulgaria

Email: emiliya@math.bas.bg

\begin{abstract}
The problem of conceptual optimization of Generalized Nets (GNs) models is discussed. An overview of some operators for complexity of GNs and relations with respect to them is presented. Some new operators and relations are defined. A GN model of a queuing system with finite capacity of the buffer and server, and FIFO discipline of service of the requests, is optimized with respect to some of the operators for complexity.
\end{abstract}

\section{INTRODUCTION}

O $\mathrm{NE}$ of the first attempts to define a set of quantifiable characteristics of a conceptual model; a measurement of the characteristics together with a fixed measurement of the decision-maker's preferences are done in Oren [8]. The proposed characteristics are: 1) size, 2) change pr. month, 3) data description inaccuracy, 4) semantic relevance, 5) semantic inaccuracy and 6) 1/0-model size. Oren mentions that the conceptual model characteristics may be quantified absolutely or relatively and discusses the accuracy of quantifying the characteristics. Examples are not given.

Another approach can be found in [10]: Conceptual modelling is about abstracting a model that is fit-for-purpose and by this we mean a model that is 1) valid, 2) credible, 3 ) feasible and 4) useful. Some important features of the conceptual models are:

- the model is designed for a specific purpose and without knowing this purpose it is impossible to create an appropriate simplification;

- simplifications are incorporated in the model to enable more rapid model development and use, and to improve transparency;

- assumptions are made either when there are uncertainties or beliefs about the real world being modelled.

The work of Velin Andonov is supported by the 2020 National Scientific Program for post doctoral and young scientists of the Ministry of Education and Science of Bulgaria. The work of Emiliya Saranova is partially supported by the joint research project "Symbolic-Numerical Decision Methods for Algebraic Systems of Equations in Perspective Telecommunication Tasks" of IMI-BAS, Bulgaria and JINR, Dubna, Russia. The work of Stoyan Poryazov was supported by the Task 1.2.5. "Prediction and Guaranteeing of the Quality of Service in Human-Cyber-Physical Systems" of National Scientific Program "Information and Communication Technologies for a Single Digital Marke in Science, Education and Security (ICT in SES)" financed by the Bulgarian Ministry of Education and Science.
The characteristics in [10] are not quantifying.

Eric [2] considers the Universe of Discourse (UoD), describing which classes of entities and propositions are important for an application area. UoD consists of: functional and existence dependencies, attributes, subtype-connections, classes, labels. Let $S$ be a concrete conceptual schema (diagram, model) which is to be evaluated by a proposed evaluation function of the following quality measures [2]:

- number of functional dependencies that hold in the UoD, but which are not expressed in $\mathrm{S}$;

- number of existence dependencies that hold in the UoD, but which are not expressed in $\mathrm{S}$;

- number of attributes and subtype connections in S;

- number of classes in $\mathrm{S}$;

- number of labels of $\mathrm{S}$.

In [6], considered Metrics for Structural Complexity are:

- number of associations - total number of associations in a model;

- number of dependencies - this metrics is used to calculate the total number of dependency relationships within the class diagram;

- number of aggregations - it calculates the number of aggregation relationships within a class diagram;

- depth inheritance tree - it calculates the longest path from the class to the root of the hierarchy in a generalization hierarchy.

Metrics for Modularity are [6]:

- Cohesion - this metric calculates the cohesion of different modules;

- Coupling - it calculates the coupling between different modules.

Many of the metrics above are difficult to be evaluated automatically. In the present paper, we use a more formal approach to metrics of structural complexity.

Generalized Nets (GNs, see [5]) are extensions of Petri Nets ([1]). For many types of Petri Nets and their extensions, it is proven that the functioning and the results of their work can be represented by an ordinary GN [4]. An important property of the GNs is that one and the same process can be modeled by more than one GN. As a result, a problem arises of choosing 
the most suitable GN model of a particular process among the many possible.

In the present paper, we study the problem for conceptual optimization of GN models. It is based on operators for complexity of GNs some of which are defined in [4], while some others are defined here for the first time. Relations of inclusion with regard to the results of the work of GNs about the operators are defined which allow a comparison of the GN models to be made. The optimization is demonstrated for a GN model of a queuing system with finite capacities of the server and buffer, and FIFO (First-In, First-Out) discipline of service of the requests. The choice of the model is justified by the fact that many GN models of queuing systems exist (see [9], [14], [15]).

\section{ON The Concepts in Generalized Net Models}

The GN is a relatively complex object. Detailed definition of a transition of a $G N, G N$ and the algorithms for transition and net functioning can be found in [5]. The concepts of a GN model can be divided into model description concepts and graphical representation concepts.

First, we shall describe non-formally the elements used in the graphical representation of a GN. GN's places are

represented by

Every transition of a GN contains transition's conditions which are graphically represented by $\mid$.

Like Petri nets, GNs contain tokens which are transferred from place to place through the arcs of the net. The arcs are denoted by arrows in Fig. 1.

The names of the transitions and the places are also included in the graphical representation of the GN model. They can be very important for the understanding of the model by nonspecialists in the area of GNs and for the users in general.

To summarize, the concepts of a GN model which are represented graphically are: transition, place, arc and the names of the transitions and the places.

\section{OPERATORS FOR COMPLEXITY OF GNS MODELS}

Some operators for complexity of GNs are defined in [4]. Below, we briefly present some of them and propose new ones. For arbitrary transition $Z$ and arbitrary GN $E$ (see [4]):

- $\phi_{1}(E)=\left|p r_{1} p r_{1} E\right|$ is the number of the transitions of the net;

- $\phi_{2}(E)=\left|p r_{1} p r_{1} p r_{1} E \cup p r_{2} p r_{1} p r_{1} E\right|$ is the number of places of the net;

- $\phi_{3}(E)=\left|p r_{1} p r_{2} E\right|$ is the number of tokens of the net;

- $\phi_{4}(E)=\left|p r_{3} p r_{3} E\right|$ is the duration of the GN functioning;

$\bullet$

$$
\phi_{5}(E)=\sum_{Z \in p r_{1} p r_{1} E} \phi_{5}^{\prime}(Z)=\sum_{k=1}^{\left|L^{\prime}\right|} \sum_{l=1}^{\left|L^{\prime \prime}\right|} k \cdot l\left(\begin{array}{c}
\left|L^{\prime}\right| \\
k
\end{array}\right)\left(\begin{array}{c}
\left|L^{\prime \prime}\right| \\
l
\end{array}\right)
$$

is operator for the complexity of the transitions of the net;

- $\phi_{6}(E)=\max _{\alpha \in p r_{1} p r_{2} E} b(\alpha)$ is the maximum number of characteristics that the tokens can keep during the functioning of the net;

- $\phi_{7}(E)=\left|p r_{1} p r_{4} E\right|$ is the number of initial characteristics of the tokens;

- $\phi_{8}(E)=\sum_{Z \in p r_{1} p r_{1} E} p r_{1} Z p r_{2} Z$ is the number of arcs of the net;

- $\phi_{9}(E)=\left|\cup_{Z \in p r_{1} p r_{1} E}\left\{l \mid l \in p r_{1} Z \& l \in p r_{2} Z\right\}\right|$ is the number of places which are both intput and output for a given transition, i.e., the number of loops. This operator gives us information about the graphical representation

- $\phi_{10}(E)=\frac{\sum_{r \in p r_{5} p r_{1} p r_{1} E}\left|\left\{r_{i, j} \mid r_{i, j} \in r \&\left(r_{i, j}=f a l s e \vee r_{i, j}=t r u e\right)\right\}\right|}{\sum_{r \in p r_{5} p r_{1} p r_{1} E}\left|\left\{r_{i, j} \mid r_{i, j} \in r\right\}\right|}$ is operator of determinacy, i.e., a ratio of the number of elements of the IMs of the predicates of the transitions with truth values "true" or "false" to the total number of predicates.

- $\phi_{11}(E)=\left|\Omega_{E}\right|$ is the number of concepts used to describe the GN $E$;

- $\phi_{12}(E)$ is the number of concepts used in the graphical representation of the GN $E$.

Above, we denote by $p r_{i} A$ the $i$-th projection of the set $A$.

If $\phi$ is some operator for complexity, then using the relations $\approx$ and $\sqsubset$ defined in [4], we can define relations of inclusion and equivalence between GNs with respect to the operator in the following way:

Definition 1: $E_{1} \vdash_{\phi} E_{2} \equiv\left(E_{1} \approx E_{2} \& \phi\left(E_{1}\right) \geq \phi\left(E_{2}\right)\right) \vee$ $\left(E_{2} \sqsubset E_{1}\right)$.

Definition 2: $E_{1} \approx_{\phi} E_{2} \equiv\left(E_{1} \approx E_{2}\right) \&\left(\phi\left(E_{1}\right) \geq \phi\left(E_{2}\right)\right)$.

\section{Optimization of A GN MOdEL OF A QUEUING SYSTEM}

Since different GNs can be used to model one and the same process, it is important to determine which one is the best with regard to the purpose of the modelling. As shown in [4], a given GN can be modified through the operators over GNs. As a result of the application of some of the operators, the resulting net can have less (or more) transitions, places, tokens, etc. Suppose we have a GN with high value of some operator for complexity $\phi_{i}$ which we want to simplify. By applying the operators to it, we can obtain a sequence of GNs $E, E_{1}, \ldots, E_{n}$ such that

$$
E \vdash_{\phi_{i}} E_{1} \vdash_{\phi_{i}} \ldots \vdash_{\phi_{i}} E_{n} .
$$

This process can continue until we obtain a GN with one transition and two places, which would be minimal with respect to the operator. However, such GN is not very useful. Therefore, this process must be terminated at some point when the last obtained GN is the most optimal one. Specifying the 


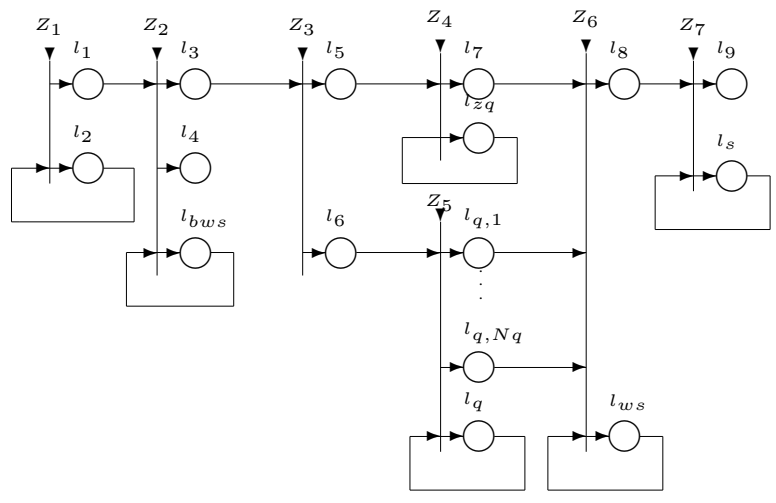

Fig. 1. First GN model of a queuing system.

optimization criteria, for example the threshold value of the corresponding operator for complexity, is a problem from the methodological aspect of the theory of the GNs. The modeller should determine the optimal number of transitions, places, tokens, etc, of the GN which give the optimal values of the complexity operator (or collection of operators).

\section{A. First GN model of a queuing system}

To illustrate the optimization of a GN, we consider a queuing system [3] consisting of buffer and server with finite capacities and FIFO (First-In, First-Out) discipline of service of the requests by the server. GN models of queuing systems with various disciplines of service of the requests are described in [14], [15]. A comparison between the GN approach to the conceptual modelling of queuing systems and the Service Systems theory approach is made in [9]. Below we use some of the results presented in these papers.

First, we propose a detailed GN model of a queuing system with graphical representation shown in Fig. 1. It corresponds to a detailed conceptual model of queuing system proposed in [9], which uses elements of Service Systems Theory.

The GN consists of 7 transitions and $14+N q$ places where $N q$ is the capacity of the buffer. The transitions represent the following functions of the queuing system:

- $Z_{1}$ represents the process of generating of requests.

- $Z_{2}$ represents the blocking of the requests when the buffer has reached its capacity.

- $Z_{3}$ determines the way of service of the requests, i.e., with waiting or without waiting.

- $Z_{4}$ represents the service of the requests without delay, when the server has not reached its capacity.

- $Z_{5}$ represents the service of the requests with waiting, when the server has reached its capacity.

- $Z_{6}$ represents the function of the buffer of the queuing system.

- $Z_{7}$ represents the function of the server of the queuing system.

A special naming system of the important places in which tokens of the GN collect the values of the parameters of the

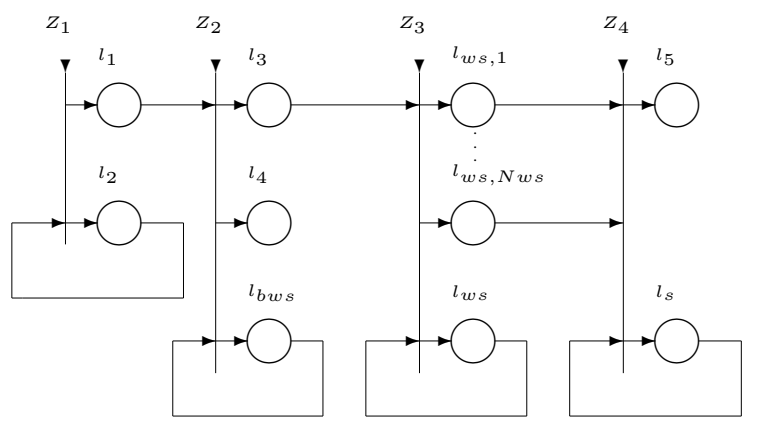

Fig. 2. Second GN model of a queuing system.

queuing system is used. Six types of tokens are used in the model.

Let $E_{1}$ be the GN shown in Fig. 1. Then $\phi_{1}\left(E_{1}\right)=7$, $\phi_{2}\left(E_{1}\right)=14+N q, \phi_{3}\left(E_{1}\right)=6, \phi_{7}\left(E_{1}\right)=6, \phi_{8}\left(E_{1}\right)=$ $24+4 N q, \phi_{9}\left(E_{1}\right)=6$,

$$
\phi_{10}\left(E_{1}\right)=\frac{17+N q}{24+4 N q},
$$

$\phi_{11}(E)=6, \phi_{12}(E)=5$.

\section{B. Second GN model of a queuing system}

The GN $E_{1}$ represents the most detailed representation of a queuing system with FIFO discipline of service of the requests. Here, we modify this model by substituting the three transitions $Z_{3}, Z_{4}$ and $Z_{5}$ with a single transition which represents the function of the buffer. The new GN is shown in Fig. 2.

Transitions $Z_{1}$ and $Z_{2}$ are the same as in the first GN model. Transitions $Z_{3}$ and $Z_{4}$ are different compared to the first GN model. Transition $Z_{3}$ represents the function of the buffer. Transition $Z_{4}$ represents the function of the server. The waiting places of the buffer are represented by places $l_{w s, 1}, l_{w s, 2}, \ldots, l_{w s, N w s}$, where $N w s$ is the buffer capacity and place $l_{w s}$ is used to store the values of the parameters of the buffer device. Four types of tokens are used in the model.

Let $E_{2}$ be the GN described above. Then we have: $\phi_{1}\left(E_{2}\right)=4, \phi_{2}\left(E_{2}\right)=8+N w s, \phi_{3}\left(E_{2}\right)=4, \phi_{7}\left(E_{2}\right)=4$, $\phi_{8}\left(E_{2}\right)=12+4 N w s, \phi_{9}\left(E_{2}\right)=4$,

$$
\phi_{10}\left(E_{2}\right)=\frac{8+2 N w s}{10+4 N w s},
$$

$\phi_{11}\left(E_{2}\right)=6, \phi_{12}\left(E_{2}\right)=5$. Since $N q=N w s$, because the buffer capacity is the same in both GNs, we obtain $E_{1} \vdash_{\phi_{i}} E_{2}$ for $i=1,2,3,7,8,9,11,12$.

\section{Third GN model of a queuing system}

In the previous two GN models, the transitions representing the function of the buffer have one place for every waiting place of the buffer. For queuing systems with low buffer capacity this is a convenient representation, especially with regard to the graphical representation. For queuing systems with large buffer capacities (or infinite) that is not optimal 


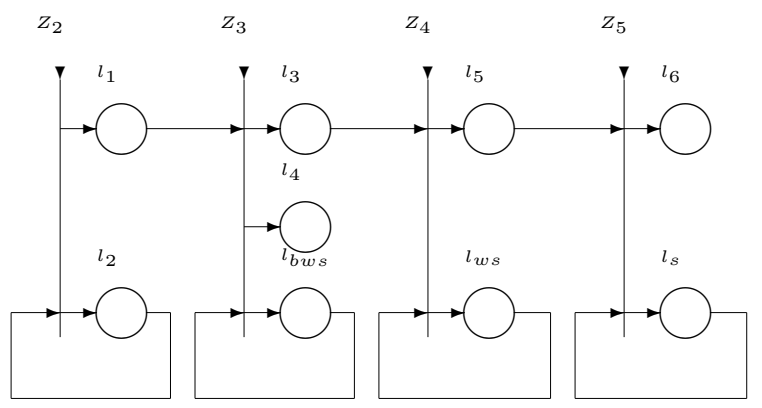

Fig. 3. Third GN model of a queuing system.

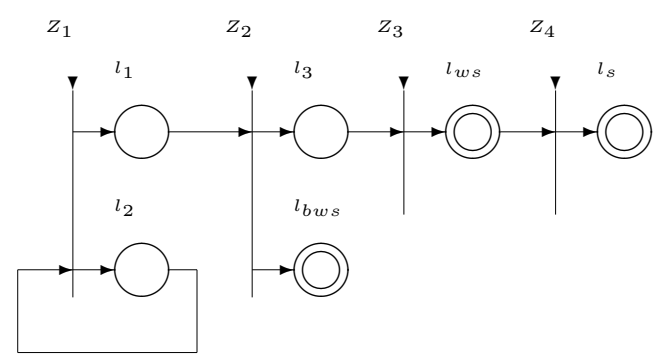

Fig. 4. Fourth GN model of a queuing system.

representation. It is possible to substitute the waiting places with one place, as in the third GN model shown in Fig. 3.

Let $E_{3}$ be the GN shown in Fig.3. The operators for complexity have the following values: $\phi_{1}\left(E_{3}\right)=4, \phi_{2}\left(E_{3}\right)=9$, $\phi_{3}\left(E_{3}\right)=4, \phi_{7}\left(E_{3}\right)=4, \phi_{8}\left(E_{3}\right)=16, \phi_{9}\left(E_{3}\right)=4$, $\phi_{10}\left(E_{3}\right)=5 / 8, \phi_{11}\left(E_{3}\right)=6, \phi_{12}\left(E_{3}\right)=5$. Therefore, we have $E_{2} \vdash_{\phi_{i}} E_{3}$ for $i=1,2,3,7,8,9,11,12$.

\section{Fourth GN model of a queuing system}

The third GN model can be further optimized if we use the extension of the ordinary GNs - Generalized Nets with Characteristics of the Places (GNCP, [11]). The places which can obtain characteristics are presented with two concentric circles in the graphical representation of the net in Fig. 4. Now, only one type of tokens is used.

Let $E_{4}$ be the GN shown in Fig. 4. Then, we have: $\phi_{1}\left(E_{4}\right)=4, \phi_{2}\left(E_{4}\right)=6, \phi_{3}\left(E_{4}\right)=1, \phi_{7}\left(E_{4}\right)=1$, $\phi_{8}\left(E_{4}\right)=6, \phi_{9}\left(E_{4}\right)=1, \phi_{10}\left(E_{4}\right)=1 / 2, \phi_{11}\left(E_{4}\right)=6$, $\phi_{12}\left(E_{4}\right)=6$. In this case, the relations $E_{3} \vdash_{\phi_{i}} E_{4}$ for $i=1,2,3,7,8,9,10,11$.

The last GN is the optimal representation of a queuing system, in the sense that it has the least acceptable number of transitions and places. It is possible to further reduce the number of transitions and places but some of the concepts of the queuing system will not be presented. The text conceptual description of a queuing system has 4 concepts which must be presented in the graphical representation of the GN model. These are: generator, blocked waiting requests branch, buffer and server. All of them are presented in the fourth GN.

\section{CONCLUSION}

The conceptual model optimization needs appropriate indicators of quality. They have to be objective and evaluated predominantly automatically, if we want to design optimization algorithms performed by computer. Most of the existing indicators are subjective. In [7], all of the 10 proposed conceptual modeling evaluation criteria are subjective. The proposed indicators here are suitable for computer evaluation.

The operators for complexity and the relations defined over GNs with respect to these operators are a base for conceptual optimization of GN models. The operators for complexity and the relations over GNs should be generalized to allow comparison of conceptual models based on the GNs theory with conceptual models in Service Systems Theory.

The comparison of conceptual models presented in different languages, e.g., comparison of conceptual models based on the GNs theory with conceptual models in Service Systems Theory is an extremely challenging task.

\section{REFERENCES}

[1] C.-A. Petri, Kommunication mit Automaten. Ph.D. Thesis, Univ. of Bonn 1962.; Schriften des Inst. fur Instrument. Math., No. 2, Bonn, 1962.

[2] C. F. Eick, A Methodology for the Design and Transformation of Conceptual Schemas, Proceedings of the 17th International Conference on Very Large Databases.Barcelona, September, 1991, 25-34.

[3] G. Giambene, Queuing Theory and Telecommunications, Springer US, 2nd Edition, 2014, https://dx.doi.org/10.5555/1205907.

[4] K. Atanassov, Generalized Nets, World Scientific, Singapore, London, 1991, http://dx.doi.org/10.1142/1357.

[5] K. Atanassov, On Generalized Nets Theory, Prof. M. Drinov Academic Publ. House, Sofia, 2007.

[6] K. Mehmood, S. Cherfi, I. Comyn-Wattiau, Data quality through conceptual model quality - reconciling researchers and practitioners through a customizable quality model. Published in ICIQ 2009 (http://mitiq.mit.edu/ICIQ/Documents/IQ\%20Conference\%202009/ Papers/2-C.pdf)

[7] M. L. Loper, L. G. Birta, G. Arbez, Lessons from a conceptual modeling exercise. Proceedings of the 2012 Winter Simulation Conference.C. Laroque, J. Himmelspach, R. Pasupathy, O. Rose, and A.M. Uhrmacher, eds. 978-1-4673-4780-8/12/ l'2012 IEEE, http://dx.doi.org/10.1109/WSC.2012.6465215

[8] O. Oren, A method for optimization of a conceptual model,1984 IEEE First International Conference on Data Engineering, Los Angeles, CA, USA, 1984, 126-132, http://dx.doi.org/10.1109/ICDE.1984.7271264

[9] S. Poryazov, V. Andonov, E. Saranova, Comparison of Four Conceptual Models of a Queuing System in Service Networks, Proc. of the 26th National conference with international participation TELECOM 2018 , Sofia, 25-26 October, 2018, 71-77.

[10] S. Robinson, Conceptual Modelling: Who Needs It?, SCS M\&S Magazine - 2010 / n2 (April)

[11] V. Andonov, K. Atanassov, Generalized nets with characteristics of the places, Compt. rend. Acad. bulg. Sci., vol 66, 12, 2013, 1673-1680.

[12] V. Andonov, Reduced generalized nets with characteristics of the arcs,Issues in Intuitionistic Fuzzy Sets and Generalized Nets, vol 14, 2018/19, 25-35.

[13] V. M. Vishnevskiy, Theoretical foundations of computer networks planning, Tehnosfera, Moscow, 2003. (in Russian)

[14] Z. Tomov, M. Krawczak, V. Andonov, E. Dimitrov, K. Atanassov, Generalized net models of queueing disciplines in finite buffer queueing systems, Proceedings of the 16th International Workshop on Generalized Nets, Sofia, 10 February, 2018, 1-9.

[15] Z. Tomov, M. Krawczak, V. Andonov, K. Atanassov, S. Simeonov, Generalized net models of queueing disciplines in finite buffer queueing systems with intuitionistic fuzzy evaluations of the tasks, Notes on Intuitionistic Fuzzy Sets, Vol 25, 2019, No 2, 115-122, https://doi.org/10.7546/nifs.2019.25.2.115-122. 